North Middlesex University Hospital developed the first joint simulation programme with some of the British Airways pilots (Project Wingman) locally.

Objectives The objectives of the joint simulation programme is to develop and improve human factors training amongst staff working the Emergency Department, by tapping on the expertise of the aviation crew in delivering key situation awareness and crisis management skills, integrating it within the relevant clinical environment so as to enhance patient care.

Methods The simulation faculty developed a clinical scenario relevant for the trainees and delivered the simulation in situ (ie within the emergency department) whilst the BA pilots who were briefed on the clinical aims of the simulation beforehand, observed the simulation and provided verbal and written feedback following the simulation. The written feedback with relatable human factor references to the aviation industry was produced but the pilot within a week following the simulation, which enabled learners continue the reflective process after the simulation at their own pace. In addition, pre and post simulation feedback was collected from the participants to better understand and improve further delivery of these simulations training events.

Results To date, Project Wingman has conducted 2 in situ simulations in the North Middlesex University Hospital with plans for further simulation dates. Attendance of the simulation involved the core simulation faculty team, BA pilots and a multidisciplinary team of clinical staff including emergency physicians, anaesthetists, nurses and operating department practitioners. Feedback from these simulations have been overwhelmingly positive, with over $90 \%$ of the participants finding it relevant and useful for their training. There is also a shift in attitude as demonstrated in the pre and post simulation feedback where hesitancy amongst participants towards the usefulness of incorporating aviation standards for providing feedback reduced.

Conclusions In conclusion, the North Middlesex University Hospital simulation faculty feel that these joint simulation programmes are beneficial to the emergency department and provides a fresh perspective towards human factors training as well as situational awareness and debriefing methods. The skills learnt from such simulation training enhances the team's ability to work cohesively as a multidisciplinary team even during highly stressful situations. Moreover, the provision of detailed written feedback following each session allows the learner to continue reflecting on the event and for the wider team who could not attend the session learn from it as well. A combined simulation programme will undoubtedly help clinicians to continue developing their skills in delivering the highest standard of patient care through challenging situations.

\section{SAFEGUARDING NON-MOBILE BABIES IN THE EMERGENCY DEPARTMENT}

Laura Duthie, Amanda King, Will Christian, Ross Goodson, Carmel Rosin, Abi Tyer. UK

10.1136/bmjpo-2021-RCPCH. 187

Background Accidental injuries in non-mobile babies are very uncommon, therefore even minor injuries could indicate serious abuse and require appropriate investigation.

In our ED in a large tertiary children's hospital in the UK, any non-mobile baby presenting with a visible injury is considered at risk of abuse and therefore follows a standardised pathway. This involves a multi-agency approach in which checks are conducted with social care and the police to ensure the clinician can make an informed decision about the safety of the child. However, audits in 2016 and 2017 demonstrated that compliance to the pathway was poor $(68.6 \%)$ with only half of clinicians completing a background check $(54.3 \%)$ or passing on these results to the GP (51.4\%).

Objectives Our aim was to identify obstacles to the non mobile baby pathway and address them in order to streamline the process for clinicians, social care and families.

Methods A multidisciplinary task group was set up including members from clinical and administration teams. With the assistance of our QI department, we completed process mapping, fishbone analysis and a prioritisation matrix. A questionnaire was then distributed amongst clinicians in order to identify specific obstacles to the pathway, and the results analysed.

Results The questionnaire was completed over two weeks in November 2020 by 27 of the medical and nurse practitioner team ranging in experience from 3 months to over 10 years.

Results identified that although all clinicians were aware of the process and $93 \%$ were able to identify patients who needed to undergo it, only $78 \%$ were confident in actually undertaking the process.

The time taken to complete the process ranged from 10 to 120 minutes amongst participants and of those who provided numerical responses, over half stated it took over 60 minutes.

Half (48\%) of respondents found it difficult to locate the phone number to call when undertaking the checks.

When asked for areas of improvement within the process, participants most commonly identified; the need for a clear guideline, easy access to phone numbers, leaflets for parents and further training for clinicians and social care.

Conclusions We believe that our process for managing injuries in non-mobile babies is fairly unique and has already improved identification of children at risk of significant harm. The feedback gained and subsequent analysis has enabled us to recognise and address specific obstacles which can be targeted for improvement, to ensure a more streamlined process that it is both time efficient and as safe as possible.

A simplified guideline and patient information leaflet has been created and approved, along with a poster for our waiting room to explain the procedure to families. We also continue to improve awareness and understanding of the procedure amongst our colleagues through education. In the near future, we also hope to integrate the procedure within our IT infrastructure to allow a more efficient pathway.

We believe that our changes enable significant improvement of the non-mobile baby pathway for both clinicians and families, in what can be a challenging and stressful process.

\section{6 EVALUATING THE SUCCESS RATES OF RADIOLOGICALLY INSERTED GASTROSTOMY, GASTROJEJUNOSTOMY AND JEJUNOSTOMIES IN PAEDIATRIC PATIENTS AT ST. GEORGE'S HOSPITAL}

Aditya Mavinkurve. UK

\subsection{6/bmjpo-2021-RCPCH.188}

Background In the past, gastrostomy tubes had to be inserted laparoscopically, however recently the trend has shifted to favour the use of percutaneous endoscopic gastrostomies, 